\title{
MAGIC highlights
}

\author{
Rubén López-Coto ${ }^{a}$ for the MAGIC Collaboration \\ Institut de Física d'Altes Energies, Spain
}

\begin{abstract}
The present generation of Imaging Air Cherenkov Telescopes (IACTs) has greatly improved our knowledge on the Very High Energy (VHE) side of our Universe. The MAGIC IACTs operate since 2004 with one telescope and since 2009 as a two telescope stereoscopic system. I will outline a few of our latest and most relevant results: the discovery of pulsed emission from the Crab pulsar at VHE, recently found to extend up to $400 \mathrm{GeV}$ and along the "bridge" of the light curve, the measurement of the Crab nebula spectrum over three decades of energy, the discovery of VHE $\gamma$-ray emission from the PWN 3C 58, the very rapid emission of IC 310, in addition to dark matter studies. The results that will be described here and the planned deep observations in the next years will pave the path for the future generation of IACTs.
\end{abstract}

\section{The MAGIC telescopes}

The two MAGIC IACTs were built and are currently operated by a collaboration of institutions in Bulgaria, Croatia, Finland, Germany, Italy, Poland, Japan, Spain, and Switzerland ${ }^{1}$.

MAGIC is located at the Roque de los Muchachos Observatory in the island La Palma (Spain). The first single telescope (MAGIC-I) started operations in 2004 and was at the time the largest IACT yet constructed ( $17 \mathrm{~m}$ diameter mirror), a fact which translated into a very low energy threshold for VHE $\gamma$-ray detection. MAGIC-I featured significant novelties in IACTs, such as the fastest sampling of Cherenkov signals (2 GSps) or active mirror control. Its ultralight carbon fiber frame and mirrors enable very fast repositioning of the telescope ( $<20$ secs for half a turn), a crucial fact to study the prompt emission of GRBs.

The introduction of a second telescope, MAGIC-II, enabled the instrument to perform stereoscopic observations with significantly better sensitivity, and angular and spectral resolutions, starting in Fall 2009. As of today the MAGIC telescopes remain the IACT array with the largest mirrors in the world. MAGIC-II was built essentially as an improved copy of MAGIC-I. The main difference between the telescopes were their cameras and their readout electronics. MAGIC I camera was composed of 577 pixels of two different sizes. On the contrary, the MAGIC II camera was built with 1039 pixels of the same size $\left(0.1^{\circ}\right)$. The readout sampling speed was 2 GSps for both telescopes, but using very different electronics.

During the summers of 2011-2012 the instrument experienced a thorough upgrade [14]: the readout was replaced by a homogeneous system based on the DRS4 analog memory sampling chip, a clone of the MAGIC-II camera and L1 trigger were installed in MAGIC-I. The upgrade of the MAGIC-I trigger results in an enlarged trigger area for the system.

\footnotetext{
ae-mail: rlopez@ifae.es

${ }^{1}$ An updated list of collaboration members can be found at http://magic.mpp.mpg.de
} 


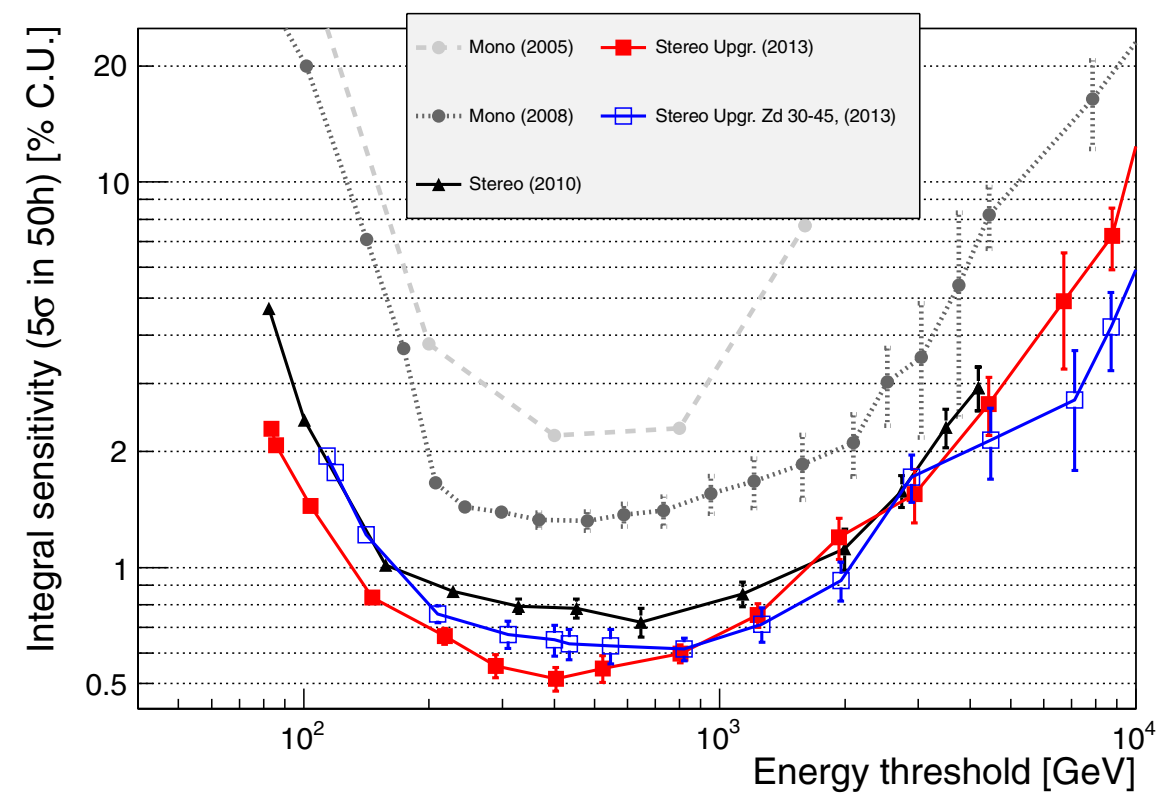

Figure 1. Integral sensitivity of the current MAGIC Stereo system and previous experimental setups, defined as the flux for which $N_{\text {excess }} / \sqrt{N_{\text {bgd }}}=5$ after $50 \mathrm{~h}$, and calculated using Crab data.

Besides, since both telescopes are now essentially identical, maintenance and operation are easier.

The trigger energy threshold (peak of the energy distribution of stereo recorded events) of the upgraded telescope has been estimated to be $50 \mathrm{GeV}$. In Fig. 1 we can see the sensitivity of the instrument as a function of energy. For energy above $300 \mathrm{GeV}$ the sensitivity is $0.76 \%$ Crab units. There is a good agreement with the predictions from MC. Respect to single telescope observations, a factor $\sim 2$ improvement in sensitivity is achieved at a few hundred $\mathrm{GeV}$ and up to a factor $\sim 3$ at lower energies. The differential sensitivity remains acceptable (10\% Crab units) below $100 \mathrm{GeV}$. Stereo observations result in a significant improvement both in angular and spectral resolutions. An angular resolution of $0.07^{\circ}$ is reached at $300 \mathrm{GeV}$. The best energy resolution of $16 \%$ is reached at a few hundred GeV. Find more details about the instrument's performance in [15].

\section{Extragalactic sources}

Radiogalaxies (active galaxies displaying a radio jet) generate CR (electron or proton) bubbles in the intergalactic medium. The same process injects magnetic field into the intracluster medium. The total injected energy is huge $\left(10^{60}-10^{61} \mathrm{erg}\right)$ : it represents a few $\%$ of the total energy of accretion into the central supermassive black hole. Relativistic electrons produce synchrotron which can be studied using radiotelescopes, but they also produce VHE $\gamma$-rays through Inverse Compton.

In fact radiogalaxies are interesting VHE sources because the emission is not so strongly beamed, i.e. the jet is not as aligned with the line of sight as in blazars, and because they are nearby objects, i.e. we can study them in more detail. IACTs have discovered four radiogalaxies at VHE: Cen-A, M 87, NGC 1275 and IC 310. MAGIC has discovered the last two sources, which actually belong to the same cluster of galaxies: Perseus. 


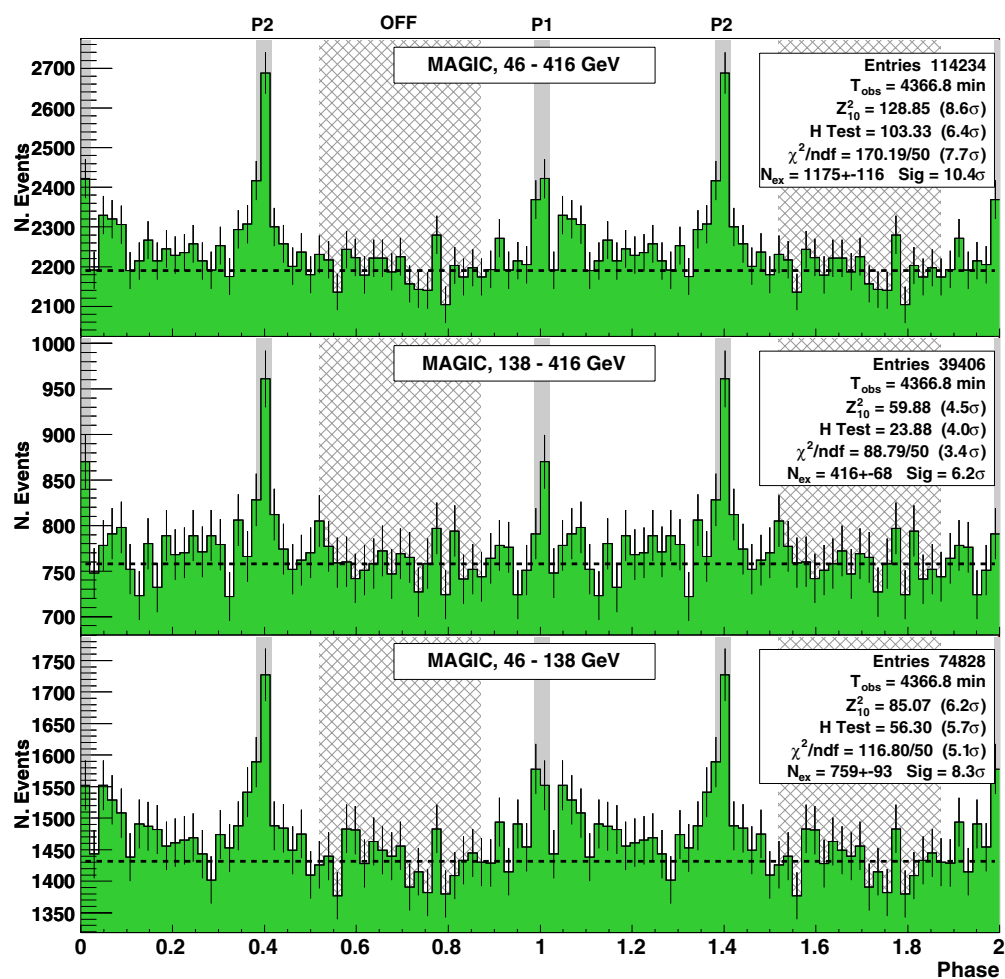

Figure 2. MAGIC folded light curves of the Crab pulsar for our total range in estimated energy and for two separate sub-bins. The shaded areas are the on-phase regions $\mathrm{P} 1_{\mathrm{M}}$ and $\mathrm{P} 2_{\mathrm{M}}$, the light shaded area is the off-region [0.52-0.87]. The dashed line is the constant background level calculated from that off-region.

MAGIC discovered the radiogalaxy IC 310 at VHE [4] during observations of Perseus. A flare was observed in 2011 which revealed that the source is variable from day to day [10]. The observation of a second flare in 2012 showed even faster variability with time scales of 1-10 minutes [12]. Even faster variability has been observed in blazars like Mrk 501 and PKS 2155-304, but emission in blazars is doppler-shifted by a larger factor than in a radiogalaxy like IC 310 for which the largest allowed Doppler factor is around 4 . The intrinsic variability of the source may in fact be so fast that the emission region is smaller than the event horizon light-crossing time. Hardly any of the existent models can accommodate such a small emission region.

S3 $0218+357$ is a blazer discovered by Fermi that in 2012 revealed a series of flares with their counterparts after $11.46 \pm 0.16$ days delay, interpreted as due to the gravitational lensing effect [18]. On 2014 July 13 and 14 Fermi-LAT detected another flaring episode, but due to the full moon period, MAGIC could not observe it. However, observations scheduled at the expected time of arrival of the gravitationally lensed component led to the first significant detection of a gravitationally lensed blazar and the most distant source detected at VHE by an IACT [23].

\section{Galactic sources}

At VHE we study the particles with the highest energy which a pulsar is able to accelerate. MAGIC discovered emission at $>25 \mathrm{GeV}$ from the Crab pulsar [16]. VERITAS discovered 


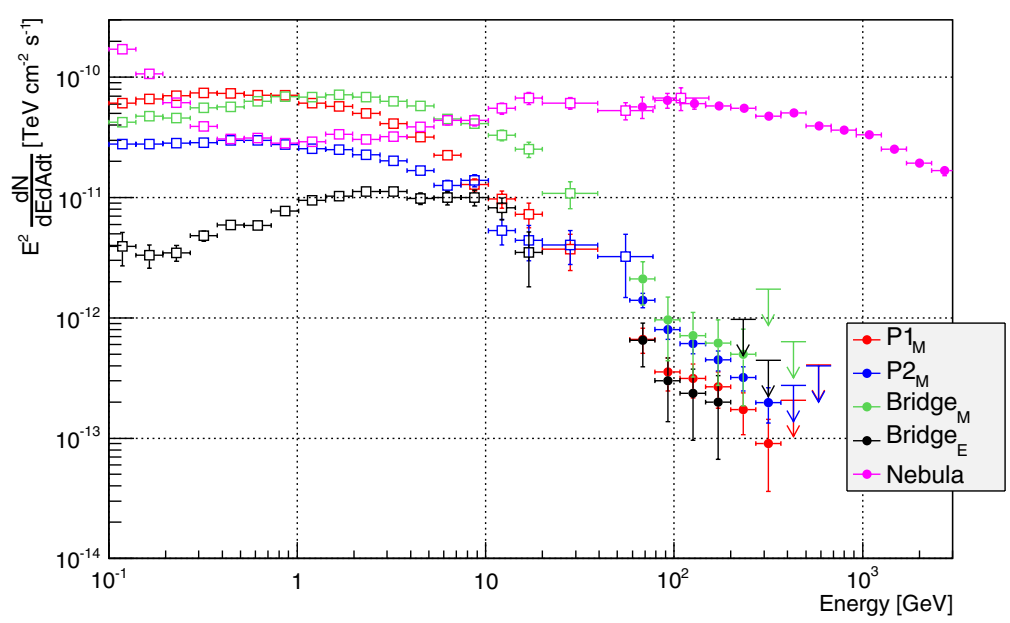

Figure 3. Spectral energy distributions of the Crab nebula, $\mathrm{P} 1_{\mathrm{M}}, \mathrm{P} 2_{\mathrm{M}}, \mathrm{Bridge}_{\mathrm{M}}$, and $\mathrm{Bridge}_{\mathrm{E}}$ measured with Fermi-LAT (below $50 \mathrm{GeV}$ ) and MAGIC (above $50 \mathrm{GeV}$ ). The flux values averaged over the rotation period are plotted.

that the pulsed spectrum extends to $>100 \mathrm{GeV}$ [17]. Some months later, MAGIC measured the spectrum of both peaks up to $400 \mathrm{GeV}$ [6] (see Figure 2). MAGIC has recently discovered emission from the "bridge" (the region between P1 and P2) of the light curve at energies exceeding $100 \mathrm{GeV}$ [8], measuring the separated spectra of both peaks and the bridge (see Fig. 3). The presence of the bridge and the fact that both peaks are very narrow is hard to explain within existing models. Aharonian et al [3] for instance propose that VHE $\gamma$-rays are not produced inside the magnetosphere but in the wind region. If true, VHE observations would allow to study the wind, which is totally dark at other wavelengths. This model is successful in producing bridge emission but predicts much broader peaks than those measured by MAGIC. Hirotani $[19,20]$ may be able to reproduce the shape of the light curve assuming that there is an additional toroidal component in the pulsar's magnetic field.

The largest population of sources in the HESS galactic plane survey are Pulsar Wind Nebulae (PWN). Energetically speaking pulsars are CR sources, that is, they spend most of their rotational power in accelerating particles. Photons, especially $\mathrm{E}<100 \mathrm{MeV}$, may well be considered as a "sideshow". MAGIC has only detected two PWN at VHE: Crab and 3C 58. These are however extreme PWNe. Crab is the brightest PWN, while 3C 58 is the weakest and least luminous. They are both the least efficient VHE PWN. 3C 58 has in fact a $\gamma$-ray luminosity which is as low as $10^{-5}$ of the pulsar spin-down power.

The latest MAGIC spectrum of the Crab Nebula is based on a 70 hour stereo observation spanning from 2009 to 2011 [13]. It extends from $50 \mathrm{GeV}$ up to $30 \mathrm{TeV}$, with a statistical precision as low as $5 \%$ at $\mathrm{E}<100 \mathrm{GeV}$ (see Figure 4). Combined with the Fermi-LAT data, these data yield the most precise measurement of the IC peak so far, at $(52.5 \pm 2.6) \mathrm{GeV}$ (statistical error only).

The spectrum has been fitted to two different models. A static, constant B-field model [22] predicts however too broad an IC peak. Most probably this implies that the assumption of the homogeneity of the magnetic field inside the nebula is incorrect. A time-dependent model [21] is successful in reproducing the spectral shape under the assumptions of a low magnetic field of less than hundred $\mu \mathrm{G}$. However, this model fails to provide a good fit of the new spectral data if the observed morphology of the nebula is taken into account. 


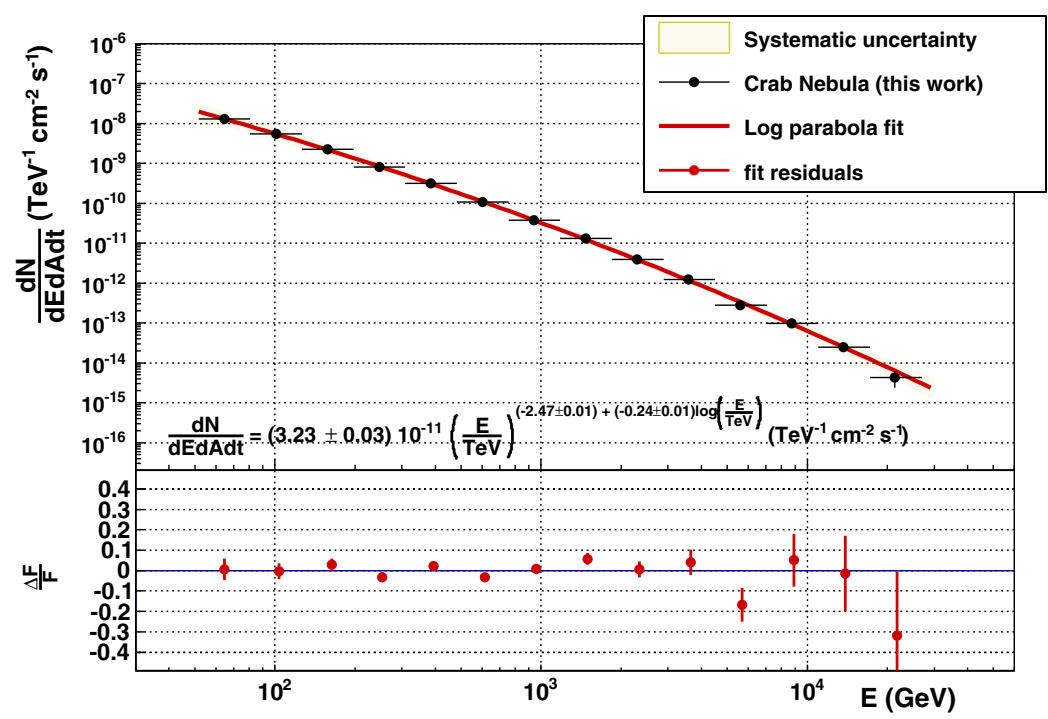

Figure 4. Differential energy spectrum of the Crab Nebula obtained with data recorded by the MAGIC stereoscopic system.

3C 58 is a PWN centered in PSR J0205+6449, one of the highest spin-down pulsars in the sky $\left(\dot{E}=2.7 \times 10^{37} \mathrm{erg} \mathrm{s}^{-1}\right.$, or $2 \%$ of the Crab pulsar's $\left.\dot{E}\right)$. The distance and age of this PWN are controversial. The distance may range between 2 and $3.2 \mathrm{kpc}$. It may be very young and associated to the historical supernova SN1181 or as old as 7000 years. The X-ray thermal emission from the central objects seems to be too weak for a neutron star in this range of ages [24], so it has been speculated that it may not be a simple neutron star, but contains a more exotic sort of matter. Like Crab it shows a torus and a jet in X-rays. Fermi-LAT detected pulsed emission at $\mathrm{E}<4 \mathrm{GeV}$ and steady emission up to $\sim 100 \mathrm{GeV}$.

MAGIC discovered 3C 58 at VHE after a $81 \mathrm{~h}$ observation [9]. Its flux is $0.65 \%$ C.U., the weakest PWN detected at these energies. For existing models, only a short distance of $2 \mathrm{kpc}$ or a high IR density can reproduce the data from radio to VHE (see Fig. 5). The IR density is probably unrealistically high, so the distance of $2 \mathrm{kpc}$ is favored. The derived magnetic field by all the models fitting the $\gamma$-ray data is in any case smaller than $35 \mu \mathrm{G}$, very far from equipartition.

\section{Dark matter}

Discovering the nature of dark matter is one of the most exciting tasks of modern science. Among the targets suitable for dark matter searches, dwarf spheroidal galaxies are considered to be excellent candidates and, among them, Segue 1 stands out with mass-to-light ratio estimated to be of the order of 1000. We present the results of stereoscopic observations of the satellite galaxy Segue 1 with the MAGIC Telescopes, carried out between 2011 and 2013. With almost 160 hours of good-quality data, this is the deepest observational campaign on any dwarf galaxy performed so far in the very high energy range of the electromagnetic spectrum. Our results represent the most stringent constraints to the annihilation cross-section or decay lifetime obtained from observations of satellite galaxies, for masses above few hundredGeV. In particular, our strongest limit (95\% confidence level), shown in Fig. 6 corresponds to a $\sim 500 \mathrm{GeV}$ dark matter particle annihilating into $\tau^{+} \tau^{-}$, and is of order $\left\langle\sigma_{\text {ann }} v\right\rangle \simeq$ $1.2 \times 10^{-24} \mathrm{~cm}^{3} \mathrm{~s}^{-1}-\mathrm{a}$ factor $\sim 40$ above the $\left\langle\sigma_{\mathrm{ann}} v\right\rangle$ thermal value [7]. 


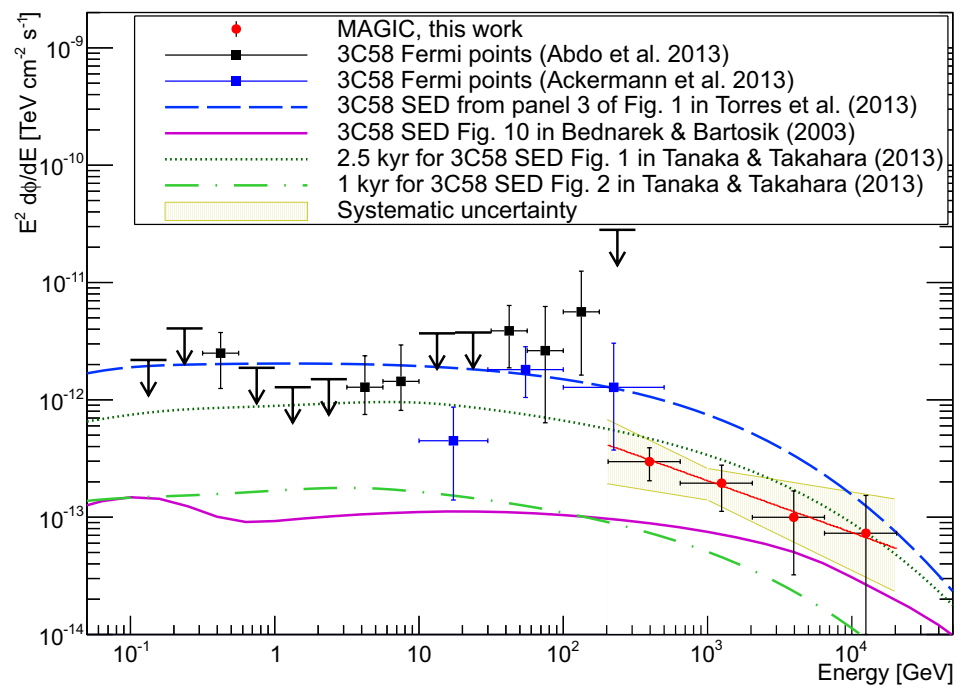

Figure 5. 3C 58 spectral energy distribution in the range between $0.1 \mathrm{GeV}$ and $20 \mathrm{TeV}$. Red circles are the VHE points reported in this work. The best-fit function is drawn in red and the systematic uncertainty is represented by the yellow shaded area. Black squares and arrows are taken from the Fermi-LAT second pulsar catalog. Blue squares are taken from the Fermi-LAT high-energy catalog. The magenta, clear green dashed-dotted, dark green and blue dashed lines correspond to different models of the source. See [9] for details.

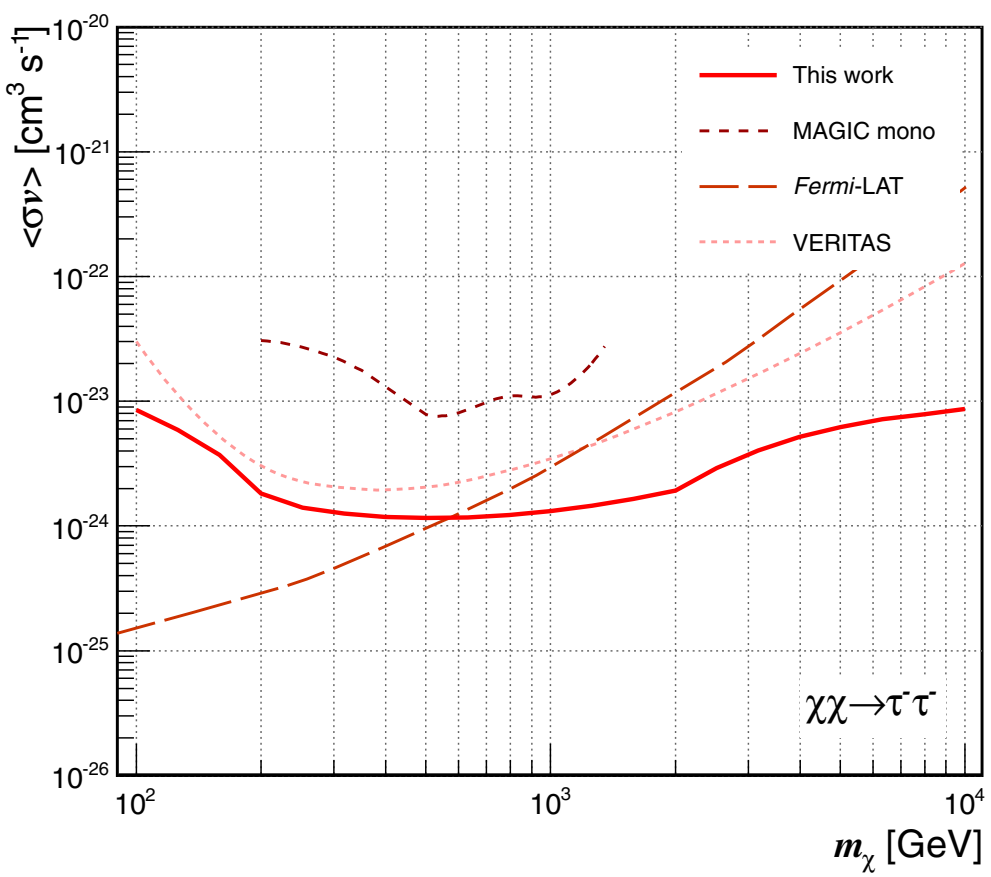

Figure 6. Upper limits on $\left\langle\sigma_{\text {ann }} v\right\rangle$ for the final state channels $\tau^{+} \tau^{-}$from the Segue 1 observations with MAGIC (solid lines), compared with the exclusion curves from Fermi-LAT (long-dashed lines), H.E.S.S. (dot-dashed line), VERITAS (dotted lines) and MAGIC-I (dashed lines). 


\section{Future prospects}

These were just a few of the latest results of MAGIC. We plan to operate the telescopes during the next few years, for sure until the first CTA telescope start to operate. Since the current generation of IACTs have already studied most of the obvious candidates for VHE emission, future observations will probably go deeper into relatively few objects. In the last years we have setup a program of Key Observation Projects to identify and observe the most promising targets.

We would like to thank the Instituto de Astrofísica de Canarias for the excellent working conditions at the Observatorio del Roque de los Muchachos in La Palma. The support of the German BMBF and MPG, the Italian INFN, the Swiss National Fund SNF, and the ERDF funds under the Spanish MINECO is gratefully acknowledged. This work was also supported by the CPAN CSD2007-00042 and MultiDark CSD2009-00064 projects of the Spanish Consolider-Ingenio 2010 programme, by grant 127740 of the Academy of Finland, by the Croatian Science Foundation (HrZZ) Project 09/176 and the University of Rijeka Project 13.12.1.3.02, by the DFG Collaborative Research Centers SFB823/C4 and SFB876/C3, and by the Polish MNiSzW grant 745/N-HESS-MAGIC/2010/0.

\section{References}

[1] A. Abramowski et al., ApJ 746, Issue 2, 151 (2012)

[2] V. A. Acciari et al., Science 325, 444 (2009)

[3] F. Aharonian et al., Nature 482, 507 (2012)

[4] J. Aleksić et al., ApJ 723, L207 (2010)

[5] J. Aleksić et al., A\&A 541, 99 (2012)

[6] J. Aleksić et al., A\&A 540, A69 (2012)

[7] J. Aleksić et al., JCAP 02, 008 (2014)

[8] J. Aleksić et al., A\&A 565, L12 (2014)

[9] J. Aleksić et al., A\&A 567, L8 (2014)

[10] J. Aleksić et al., A\&A 563, A91 (2014)

[11] J. Aleksić et al., A\&A 567, 15 (2014)

[12] J. Aleksić et al., submitted for publication

[13] J. Aleksić et al., submitted to JHEAp and arXiv:1406.6892

[14] J. Aleksić et al., submitted to Astroparticle Physics, arXiv:1409.6073

[15] J. Aleksić et al., submitted to Astroparticle Physics, arXiv:1409.5594

[16] E. Aliú et al., Science 322, 1221 (2008)

[17] E. Aliú et al., Science 334, 69 (2011)

[18] Cheung, T. et al., ApJ 782, L14 (2014)

[19] K. Hirotani, ApJ 733, L49 (2011)

[20] K. Hirotani, ApJ 766, 98 (2013)

[21] J. Martin, D. F. Torres, N. Rea, MNRAS 427, 415 (2012)

[22] M. Meyer, D. Horns, H. Zechlin, A\&A 523, A2 (2010)

[23] R. Mirzoyan et al., ATel 6349

[24] P. Slane et al., ApJ 571, L45 (2002) 